\title{
Lightweight Frame Scrambling Mechanisms for End-to-End Privacy in Edge Smart Surveillance
}

\author{
Alem Fitwi ${ }^{\dagger}$, Yu Chen ${ }^{\dagger}$, Sencun $\mathrm{Zhu}^{\ddagger}$ \\ ${ }^{\dagger}$ Dept. of Electrical and Computer Engineering, Binghamton University, Binghamton, NY 13902, USA \\ ${ }^{\ddagger}$ Dept. of Computer Science and Engineering, Penn State University, University Park, PA 16802, USA \\ Emails: \{afitwil, ychen\}@binghamton.edu, sxz160@psu.edu
}

\begin{abstract}
As smart surveillance becomes popular in today's smart cities, millions of closed circuit television (CCTV) cameras are ubiquitously deployed that collect huge amount of visual information. All these raw visual data are often transported over a public network to distant video analytic centers. This increases the risk of interception and the spill of individuals' information into the wider cyberspace that causes privacy breaches. The edge computing paradigm allows the enforcement of privacy protection mechanisms at the point where the video frames are created. Nonetheless, existing cryptographic schemes are computationally unaffordable at the resource constrained network edge. Based on chaotic methods we propose three lightweight end-to-end (E2E) privacy-protection mechanisms: (1) a Dynamic Chaotic Image Enciphering (DyCIE) scheme that can run in real time at the edge; (2) a lightweight Regions of Interest (RoI) Masking (RoI-Mask) scheme that ensures the privacy of sensitive attributes on video frames; and (3) a novel lightweight Sinusoidal Chaotic Map (SCM) as a robust and efficient solution for enciphering frames at edge cameras. Design rationales are discussed and extensive experimental analyses substantiate the feasibility and security of the proposed schemes.
\end{abstract}

Keywords-Chaotic Methods, Edge Computing, PrivacyPreserving, Video Scrambling, Smart Surveillance.

\section{INTRODUCTION}

The rapid advancement and proliferation of electronic technologies in recent years have driven urban areas to become a lot smarter. Currently, a multitude of cities around the world employ a spectrum of information and communication technologies (ICT) and Internet of Things (IoT) to improve the quality of urban services and to ensure the safety and security of their residences [4]. Surveillance is often practiced through the use of both fixedly deployed closed circuit television (CCTV) cameras and cameras mounted on mobile manned or unmanned aerial and ground vehicles like airplanes, satellites, drones, manned ground patrolling vehicles, and unmanned ground vehicles (UGV) [5], [12]. The ubiquitous and versatile deployment of these surveillance cameras in public places including streets, city corners, stores, and marketplaces enable the first-responders, government agencies, or security service providers to garner a great deal of audiovisual information about many individuals indiscriminately without their knowledge and consent [3], [8], [17], [23].

Up to 2020, there are about 770 millions of fixed surveillance cameras in operation in urban and suburban areas across the world [20]. All the visual information about many individuals created and collected by these CCTV cameras, which serve as the eyes and ears of the video surveillance system (VSS), are most often transported over a public network to distant video analytic and surveillance operation centers (SOC). This is one of the major factors that increase the likelihood of the breach of individuals' privacy due to some inherent vulnerabilities of the network architecture. The TCP/IP network architecture, widely used in today's Internet, is liable to many threats for it was initially designed without due sufficient concern to security [15]; it is one of the most ingenious human inventions of the $20^{\text {th }}$ century, though. Hence, any deployment of the surveillance system without due attention to such flaws is likely to increase the risk of privacy invasion. Most of today's VSS are deployed based on either the fog or cloud computing architectures. The cloud computing paradigms get the video analytics performed in remote cloud centers that have tremendous computational capability. However, the raw video streams may be intercepted exploiting the vulnerabilities of the network over which they are channeled. Generally, the fog and cloud computing paradigms increase the probability of privacy breaches.

Meanwhile, the edge computing paradigm migrates some intelligence and computational prowess to the smart cameras allowing End-to-End (E2E) privacy-preserving mechanisms to be enforced at the point where the videos are created. E2E Privacy refers to the scenario where information between two or more communicating parties or end systems is exchanged over secure communication channels that prevent unauthorized parts from accessing the information. In the case of a VSS, the video frames are supposed to be scrambled or encrypted on edge cameras before transmission in order to ensure that only legitimate recipients or devices that hold the unscrambling key are able to reverse the frames. This decreases the chance of divulging of individuals' data into the wider cyberspace where there are more than 4.5 billion users [6]. However, edge computing is a resource-constrained environment and it cannot support most of the well-known encryption methods that are compute-intensive and computer-intensive. Lightweight techniques that balance the privacy preserving requirements and affordable processing complexity are compelling [11].

In this work, we introduced and experimentally validated four lightweight video frame scrambling schemes. They are affordable to edge CCTV cameras to enable E2E privacy in video surveillance systems (VSS). The major contributions of this paper are summarized as ensues:

- To further improve the processing speed, a simpler 
but non-linear lightweight Dynamic Chaotic Image Enciphering (DyCIE) scheme is introduced based on a discrete chaotic dynamic system [7], [22], [28], which can run at the edge of the network in real time. A number of control parameters are introduced ensuing a thorough security analysis. The DyCIE scheme is highly sensitive to slight changes in the initial conditions that are used as parts of the key. As a result, DyCIE is more efficient for video encryption in terms of both speed and security.

- Based on the Peter De Jong Map [14], a lightweight Regions of Interest (RoI) Masking (RoI-Mask) scheme is proposed to ensure the privacy of sensitive attributes on video frames. As the original Peter de Jong map is prone to a range of attacks, we introduce additional control parameters along with a lot of experimental and fine-tuning works to transform it into a form usable for cryptographic purposes.

- Inspired by logistic map [7], [22], [28], a wellknown low-dimension chaotic system, a novel lightweight Sinusoidal Chaotic Map (SCM) is proposed for faster full video-frame scrambling. SCM is a more robust and efficient solution for enciphering frames at edge cameras.

- In order to verify the computational efficacy and security of the proposed schemes, comprehensive experimental study and analysis have been carried out, including computational performance analysis, standard security analyses, and comparative analyses with existing equivalent methods. The results solidly corroborate the feasibility of the proposed schemes.

The rest of the paper is organized as follows. Section II discusses the related works on video privacy protection schemes and chaotic theories. Section III presents a high-level architecture for E2E privacy-preserving scheme in the practice of video surveillance. Section IV elucidates the bolts and nuts of the computationally-thin DyCIE scheme developed based on a simple logistic map. Section V presents the RoI-Mask scheme for masking RoIs on video frames. The SCM scheme for full-frame scrambling is explicated in Section VI ensued by the presentation of a frame-shuffling scheme in Section VII. The detailed experimental analyses and results are presented in Section VIII. At last, the conclusions are presented in Section IX.

\section{RELATED WORKS}

\section{A. Video Privacy-protection Techniques}

In general, today's image privacy protection schemes can be put into four categories, namely editing, face regions, false color, and JPEG [30]. However, apart from the encryption scheme, most of the editing schemes are unable to completely hide sensitive contents on images. Besides, they are prone to reconstruction attacks [26], [34]. They include simple schemes like blurring, black box, pixelation, and masking. But most importantly, information is not fully recoverable. Similarly, the face regions approach [16], [23], [31] suffers from not being appropriate to real-time processing and reversibility. The false color [32] and JPEG [1], [38] methods also suffer from similar type of problems. They fail to meet the requirements set to achieve a good trade-off between usability and privacy.

Ideally, video-contents privacy protection schemes must accomplish a good balance among privacy, clarity, reversibility, security, and robustness [27], [29]. Privacy refers to the condition of not being identified by human observers without one's knowledge and consent. Clarity is an important requirement that any privacy protection mechanism should allow the identification of suspicious behavioral patterns. Any privacy-protection scheme should have a reversibility attribute in order for privacy-protected frames that contain crime scenes or criminal activities to be reversible. In addition, the scheme must be secure and robust. The security property ensures that the encrypted video frames are reversed only by authorized parties and the robustness property guarantees that the scheme does not fail to detect sensitive features or classify frames accurately.

Encryption is a member of the editing class privacy protection schemes. Given the aforementioned weaknesses and requirements, encryption/scrambling is the most secure video privacy protection scheme compared to the other editing schemes. It protects private information and sensitive data on video frames from unauthorized accesses by scrambling sensitive features before transmission. This way, it ensures E2E privacy and security of the communication between two communicating parties. There are plenty of encryption schemes today; however, most of them are not suitable for encrypting real-time video frames. Given the real-time nature and bulkiness of videos, public-key cryptographic schemes like Rivest, Shamir and Adleman (RSA) and Elliptical Curve Cryptography (ECC) are too slow to be employed in such scenarios. The traditional symmetric key cryptographic mechanisms like triple Data Encryption Standard (3DES), International Data Encryption Algorithm (IDEA), the malleable and fast Rivest Cipher 4 (RC4) and Advanced Data Encryption chaining block cipher (AES-CBC) are not convenient for image enciphering. AES is considered as one of the most secure ciphers commonly used in the secure sockets layer (SSL) or transport layer security (TLS) across the Internet today. However, it is relatively slow to be employed for real-time video encryption at the edge, where there is a limited computational power. RC4 is a quick stream cipher but it is not considered as a secure cipher any longer because of its vulnerability to a bit-flipping attack where one in every 256 keys can be a weak key. In addition, as shown by the previous studies [2], [35] as well as by our own evaluation in Section VIII-E, both AES and RC4 are not good in breaking the strong correlation amongst horizontally, vertically, and diagonally adjacent pixels of video frames.

The most popular schemes for image encryption are those mechanisms created based on chaotic schemes because of their better performance and high security. Unlike AES, the chaotic schemes can break the strong correlation amongst the pixel values of information-rich images/frames. There are chaotic maps of different dimensions including one dimensional, multidimensional and cascaded chaotic systems. There are many good chaotic systems proposed by a number of researchers [13], [19], [28], [40], [41]. More recently proposed 
chaotic image enciphering mechanisms like [21], [33] are robust. However, they are still compute-intensive and cannot achieve the required performance in resource-constrained environments, like the edge of a network. They must be customized to fit into edge devices; or new lighter methods must be introduced. Hence, this work aims at proposing novel lightweight video-frame scrambling schemes for secure and efficient enciphering of video frames at the edge of networks to ensure E2E privacy vis-à-vis the practice of VSS.

\section{B. Chaotic Theory}

Literally, a chaos is a state of complete disorder and confusion. In chaos theory, a chaotic system is a dynamical system that is highly sensitive to initial conditions, and one which is topologically mixing. Chaos can be produced either by using uncontrolled or controlled systems, as portrayed in Fig. 1. The ones in Fig. 1(a) are produced by uncontrolled natural processes. The top one is a picture of the turbulence in the tip vortex from an airplane wing and the bottom one is a random fractal of lightning scene. Figure 1(b) illustrates a 10-scroll chaotic-attractor produced by a controlled chaotic generator that we worked on.

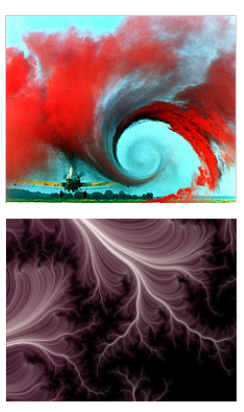

(a)

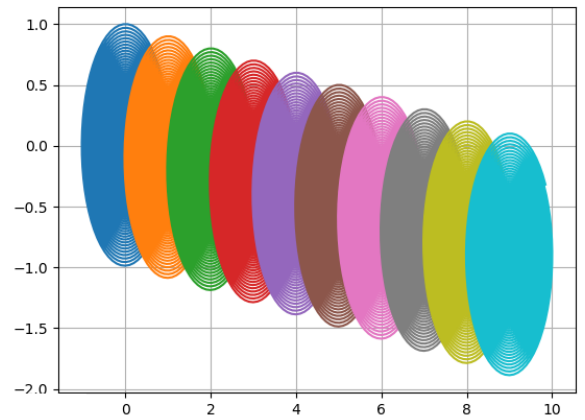

(b)
Fig. 1. Chaos: (a) chaoses produced by uncontrolled natural processes [18], (b) a chaos produced by a controlled system.

Chaotic techniques are the most useful ones in video frames/images scrambling because they have better performance, high degree of sensitivity to slight changes in initial conditions, higher degree of randomness, enormous key space, and high security. Particularly, they are able to dissociate the strong correlation among the adjacent pixels of a frame. Besides, a video frame/image contains bulky information; as a result, the traditional methods are slow to be used to encrypt such bulky data in a time sensitive application. Another most outstanding advantage of employing chaos for scrambling video frames in lieu of other methods is the fact that it can be easily vectorized. The vectorization process is employed to speed up the implementation of bulky data represented in the form of $2 \mathrm{D}$ or $3 \mathrm{D}$ matrices or rank 2 or 3 tensors, as portrayed in Fig. 2, without using a computationally expensive looping structure. Once the chaos is produced, its 3D matrix of pixel values, shown in Fig. 2, can be matched with the corresponding pixels of the frame and operated in parallel. Using such an approach can drastically improve the computational performance.

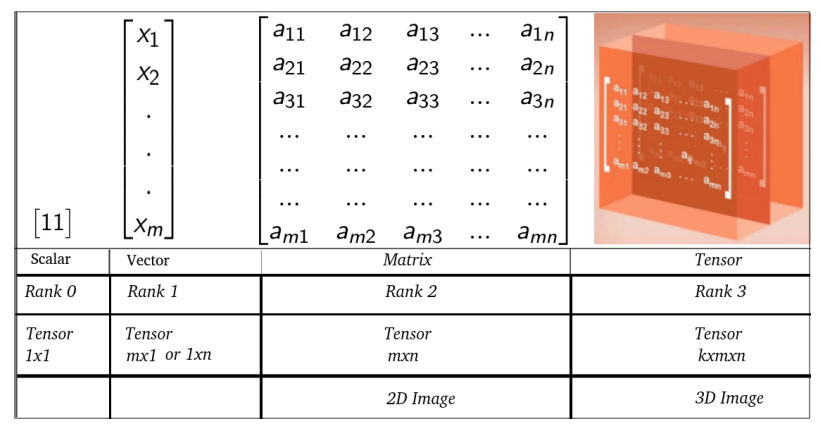

Fig. 2. Tensors of various Ranks.

Eq. (1) illustrates the vectorized bit-wise-xor operation between two RGB colored images (a chaos and video frame), whose height, width, and depth are given to be $H, W$, and $M$, respectively. Putting it another way, the pixels in corresponding locations are operated in parallel unlike the serial bit-wise xor operation where only a pair of corresponding pixels are operated at a time.

$I_{1} \oplus I_{2}=\sum_{i=0}^{W-1} \sum_{j=0}^{H-1} \sum_{k=0}^{M-1} I_{1}(i, j, k) \oplus \sum_{i=0}^{W-1} \sum_{j=0}^{H-1} \sum_{k=0}^{M-1} I_{2}(i, j, k)$

TABLE I

COMParing Serial and Vectorized Bitwise-Xor Operator

\begin{tabular}{|l|l|l|}
\hline Parameter & $\begin{array}{l}\text { Serial } \\
\text { Bitwise-xor }\end{array}$ & Vectorized-xor \\
\hline Time (ms) & 456.863 & 2.992 \\
\hline Relative Performance & $\begin{array}{l}0.00654 \text { times } \\
\text { as fast as the } \\
\text { vectorized xor }\end{array}$ & $\begin{array}{l}152.7 \text { times } \\
\text { faster than the } \\
\text { serial xor }\end{array}$ \\
\hline
\end{tabular}

Table I compares the difference in performance between serial and vectorized bit-wise-xor operations. Two 3-channel colored 480P $(640 \times 480 \times 3)$ frames/images were employed for the experiment. The results in Table I show that the vectorized operator is about 153 times faster than its serial version. This, along the good security properties, lays a strong rationale for employing chaotic schemes for image scrambling to ensure E2E privacy. However, most preexisting chaotic schemes suffer from higher computational complexity. They are not suitable for deployment in edge cameras which have very limited computational resources. This work, therefore, focuses on designing, testing, and implementing lightweight and secure chaotic methods for video frames scrambling. In our earlier work, a computationally-thin 2D Reproducible Chaotic (2D-ReC) scheme for full video frame scrambling was proposed [10]. It scrambles RGB video frames channelwise to ensure E2E privacy. It is designed to be more suitable for video frame enciphering on edge CCTV camera than existing traditional data encryption and chaotic schemes. In the experimental results of this paper, the $2 \mathrm{D}-\mathrm{ReC}$ scheme is also included for a comprehensive comparison study. 


\section{Privacy Preserving Smart Edge Surveillance}

Figure 3 shows a high level overview of the architecture of the smart VSS at the edge, in which our proposed videoframe scrambling mechanisms are envisioned for ensuring E2E privacy. There are at least five actors, namely edge camera, fog/cloud server, storage site, SOC, and law enforcer, involved in the current VSS architecture. Hence, the E2E privacy ensures that no one can eavesdrop on the contents of video frames while they are in transit. No adversary can snoop on the video frames without authorized permissions. This helps prevent the breaches of individuals' privacy by wiretapping or interception. Cryptographic methods are, therefore, employed to ensure E2E privacy. In other words, video frame encryption is of paramount importance for ensuring the E2E protection of privacy in VSS. More detailed discussions on the design rationales, function blocks, and evaluation reports of the edge VSS is beyond the scope of this paper, interested readers can find details in literature [9], [10], [11], [24], [25], [36], [37]. This paper is about mechanisms that could be employed to ensure privacy in VSS, not about the surveillance per se.

Privacy-protection schemes must be secure and robust. It must ensure that the reversion is done only by authorized parties who possess the right set of keys. A robust but lightweight key distribution management scheme is vital specifically in our E2E privacy-preserving schemes. Therefore, a simplified key management has been introduced. As portrayed in Fig. 3, video streams are transmitted from cameras to the fog/cloud server, from the fog/cloud server to the viewing stations, or from the fog/cloud server to storage locations in encrypted form. Hence, a client-server architecture based lightweight agents are employed for efficient key distribution. The key is generated in the form of list data structure, $K e y=\left[K_{R}, K_{G}, K_{B}\right]$, where $K_{R}, K_{G}$ and $K_{B}$ are the set of keys employed for the enciphering of the three channels of the input frame. Camera, server, storage site, and viewing-station agents are developed and deployed.

The camera agent stores the public key of the server agent, and the server agent keeps record of the public keys of the viewing-station and storage agents for the purpose of secure session key exchange. The viewing-station agent unscrambles frames to be viewed live by security personnel in a SOC.

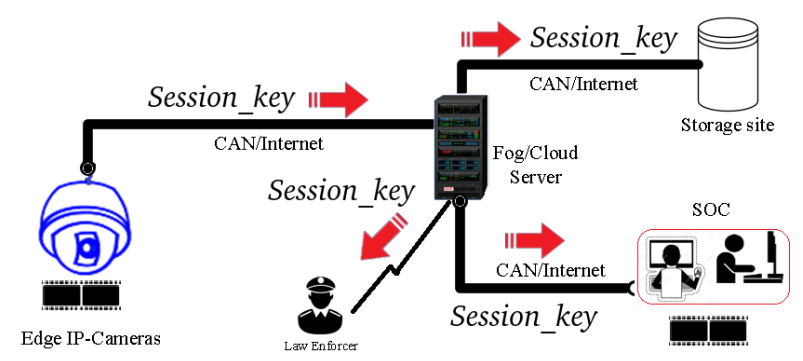

Fig. 3. Simplified End-to-End Privacy Diagram of Agents Interaction for key exchange.

\section{Dynamic Chaotic Image Enciphering Scheme}

In this section, a lightweight dynamic chaotic image enciphering (DyCIE) scheme is introduced as an Improved
Logistic Map (ILM). Developed after thoroughly investigating simpler discrete chaotic dynamic systems [7], [22], [28], DyCIE scheme can run at the edge in real-time. It is highly sensitive to any slight variation in the values of a key and more efficient for video encryption. The key is defined as a list data type in Eq. (2) and its elements constitute the coefficients and initial value of the chaotic dynamic system.

$$
\begin{aligned}
K & =\left[k_{0}, k_{1}, k_{2}, k_{3}, k_{4}\right] \\
C_{0} & =k_{0} \\
t m p & =k_{1} * C_{0}\left(1+k_{2} * C_{0}\right) \\
C & =t m p * k_{3} \text { (scaling) } \\
C_{0} & =t m p * k_{4} \text { (update) }
\end{aligned}
$$

where $k_{0} \in(0.2,0.9), k_{1} \in(3.89,4.0), k_{2} \in(-1,-0.989)$, $k_{3} \in(254,255)$, and $k_{4} \in(0.99,1)$.

Eq. (2) is recursive in that the new chaotic values of the dynamic system in the equation are generated based on previous values multiplied by some control parameters. It starts with an initial value $C_{0}$ and coefficients $k_{1}$ and $k_{2}$. Then, it is iterated until a maximum number of iterations equal to the product of the height and width of an input frame is reached. Extensive experiments and analyses have been carried out to obtain the secure ranges of the initial value $k_{0}$, and the coefficients $k_{1}, k_{2}, k_{3}$, and $k_{4}$, which are generated in the form of encryption key. Based on our experimental study, $k_{0}$ can take any double-precision floating value between $(0.2,0.9)$. For $k_{1}$, the secure range that generates secure random chaos is found out to be between $(3.89,4.0) . k_{2}$ and $k_{4}$ can assume floating values in the range of $(0.99,1.0)$ and $k_{3}$ must be within the range of $(254,255)$.

As outlined by Algorithm 1, the DyCIE scheme comprises three major components: key generator, chaos generator, and frame scrambler. The key is defined as a list data type whose elements are the initial value and the coefficients of the enciphering equations. Their values fall within the defined secure ranges and are generated using a secure cryptographic random generator. The chaos generator, which takes the key and the current video frame dimensions as inputs, produces a random chaos that in turn is used as a key to encipher frames.

To enhance the security of DyCIE scheme and to cut down on its computational complexity, the enciphering process is performed channel-wise. As portrayed in Fig. 4, the frame to be encrypted is efficiently split into the three color channels, namely red $(\mathrm{R})$, green $(\mathrm{G})$, and blue $(\mathrm{B})$. Then, the three color channels are encrypted in parallel and the results are stacked up together before transmission. The three keys are also appended into a single list before transmission in the same order as the color channels (RGB). Figure 4 illustrates that the enciphering process is performed at the edge and the inverse process is performed at the fog/cloud layer as shown in Section III.

\section{Regions of InTEREST MASKING}

Unlike the DyCIE methods, the lightweight Regions of Interest Masking (RoI-Mask) scheme was developed based on the Peter De Jong Map [14] specifically for minor's privacy protection [39]. The Peter De Jong map is a type of 2D 


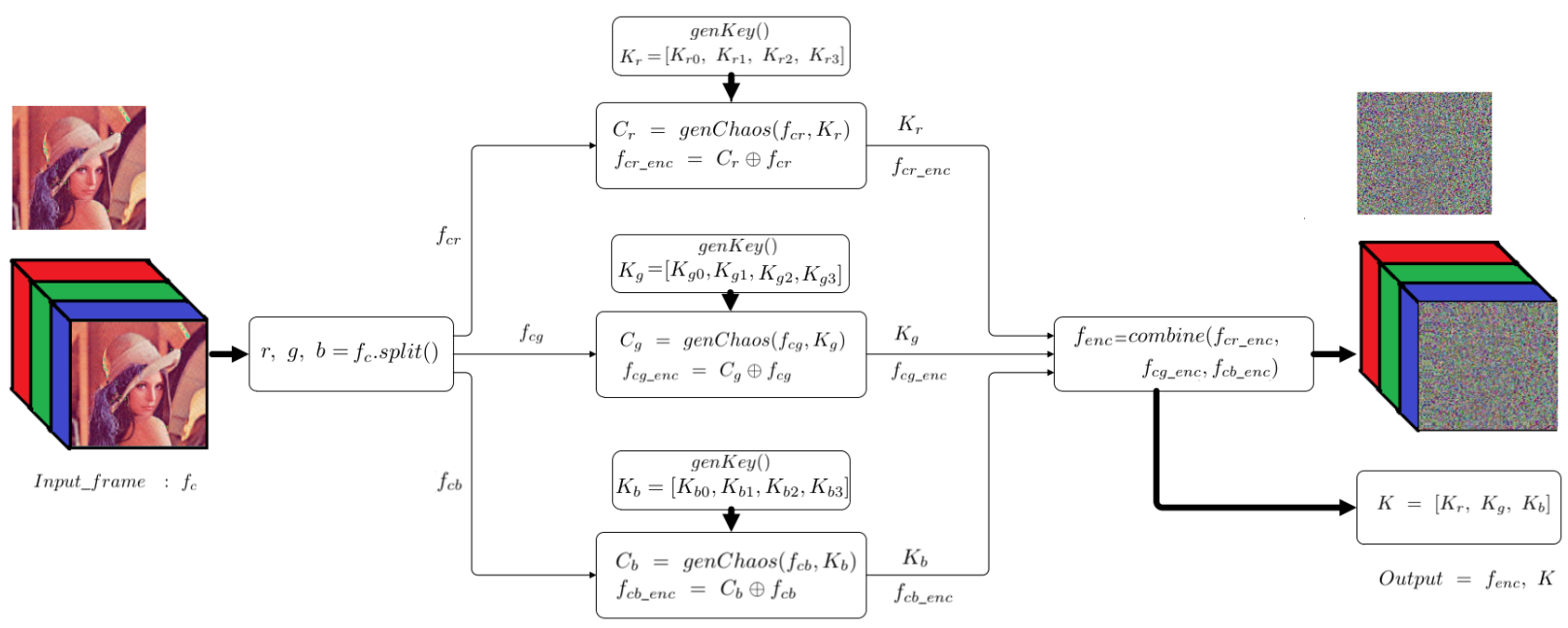

Fig. 4. Channel-wise Frame Enciphering: Color channels R, G, and B are scrambled in parallel in order to expedite the scrambling process.

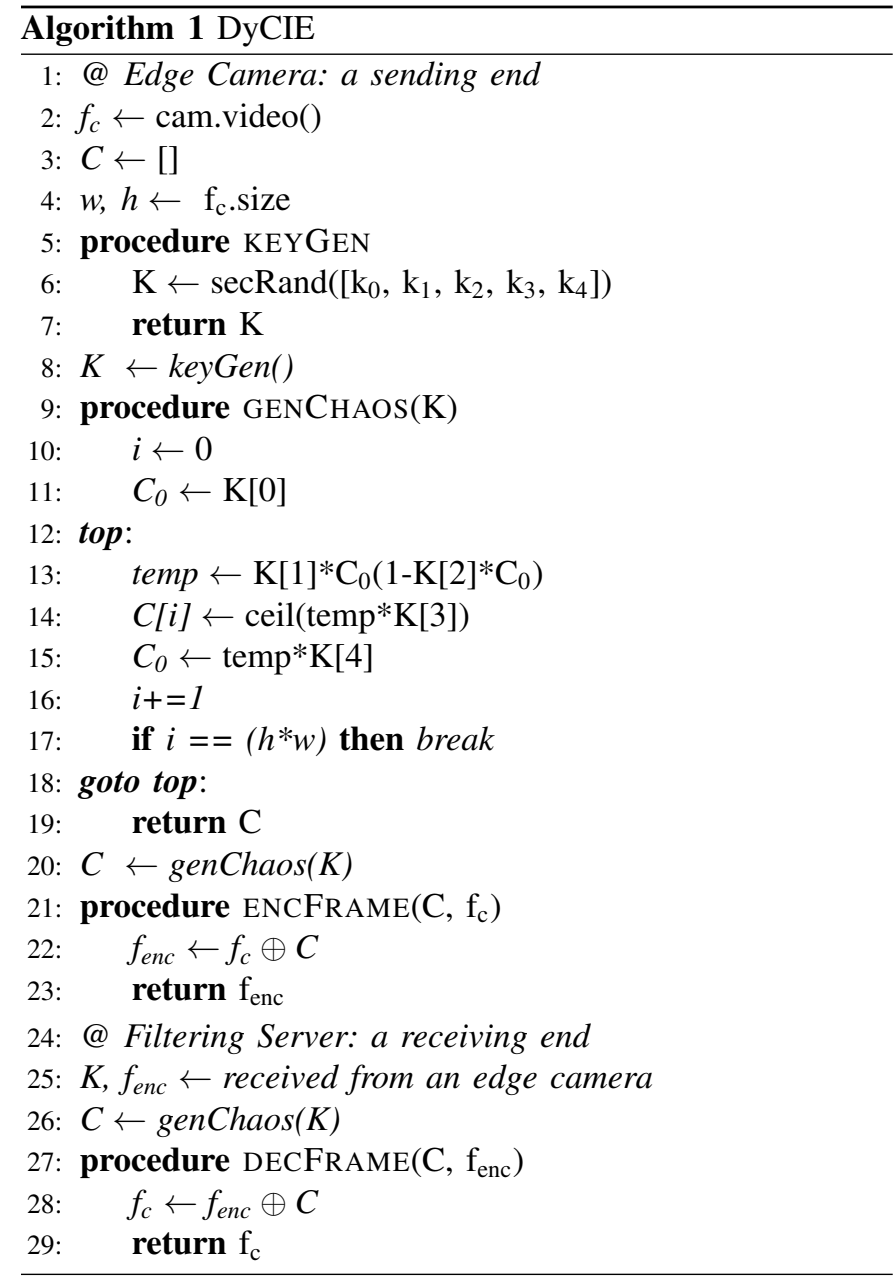

recursive system stated in Eq. (3). The choice of parameter values and initial conditions will generate totally different sets of attractors. It has four parameters and two initial conditions. However, the Peter de Jong map cannot be used for chaotic encryption as it is. It does not meet the security requirements. For instance, Fig. 5 shows a non-uniform distribution of the pixels of chaos generated by Eq. (3), signifying that it is glaringly insecure.

$$
\begin{aligned}
& x_{n+1}=\sin \left(a \times y_{n}\right)-\cos \left(b \times x_{n}\right) \\
& y_{n+1}=\sin \left(c \times x_{n}\right)-\cos \left(d \times y_{n}\right)
\end{aligned}
$$

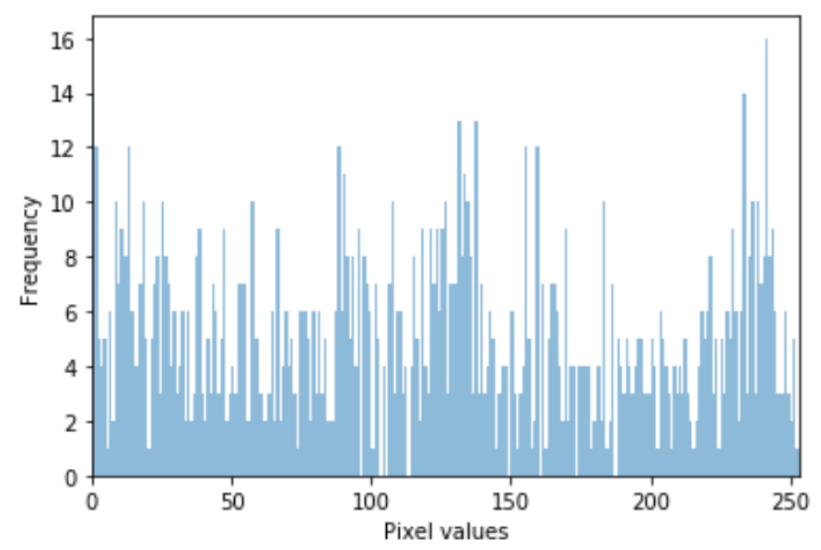

Fig. 5. Distribution of the De Jong Chaos: it is not uniform revealing its weakness against histogram analysis attack.

Following an extensive experimental study, RoI-Mask is proposed as a one-way scrambling of regions of interest in video frames using an improved version of De Jong map (IDJM) and vectorized pixel-array multiplication. As illustrated in Eq. (4), four more parameters are added and their secure ranges are identified. It generates a random and uniform output that is secure to be used for cryptographic purpose. The generated random chaotic sequence passes all standard security tests like entropy and statistical analyses. Besides, it has a key space far greater than the lower secure key space boundary (128 bits). The key contains eight double-precision floating value elements giving a key length of $64 \times 8=512$ bits and a key space of $2^{512}$.

Algorithm 2 shows the RoI-Mask algorithm. A key comprising eight elements is first generated followed by a random chaos used to scramble the RoIs on video frames. 
The top five lines of Eq. (4) illustrate the secure range of the eight elements of the key employed to generate a chaos equal to the size of the RoI. Every element of the key is a double-precision floating-point value randomly selected from its respective range, which were identified after an extensive experimental and security analysis. These values allow to produce secure chaotic outcomes that are of the same size as the ROI, and a vectorized point-wise multiplication operation is performed, which results in secure cipher.

$$
\begin{aligned}
k e y & =\left[k_{0}, k_{1}, k_{2}, k_{3}, k_{4}, k_{5}, k_{6}, k_{7}\right. \\
k_{0}, k_{1} & =\operatorname{random}(1,4), \operatorname{random}(1,4) \\
k_{2}, k_{5} & =\operatorname{random}(-1,1), \operatorname{random}(-1,1) \\
k_{3}, k_{6} & =\operatorname{random}(-1,-0.99), \operatorname{random}(-1,-0.99) \\
k_{4}, k_{7} & =\operatorname{random}(-3,-3), \operatorname{random}(-3,-3) \\
x_{0}, y_{0} & =k_{0}, k_{1} \\
x_{1} & =\sin \left(k_{2} * y_{0}\right)+k_{3} * \cos \left(k_{4} * x_{0}\right) \\
y_{1} & =\sin \left(k_{5} * x_{0}\right)+k_{6} * \cos \left(k_{7} * y_{0}\right) \\
x_{0}, y_{0} & =x_{1}, y_{1}
\end{aligned}
$$

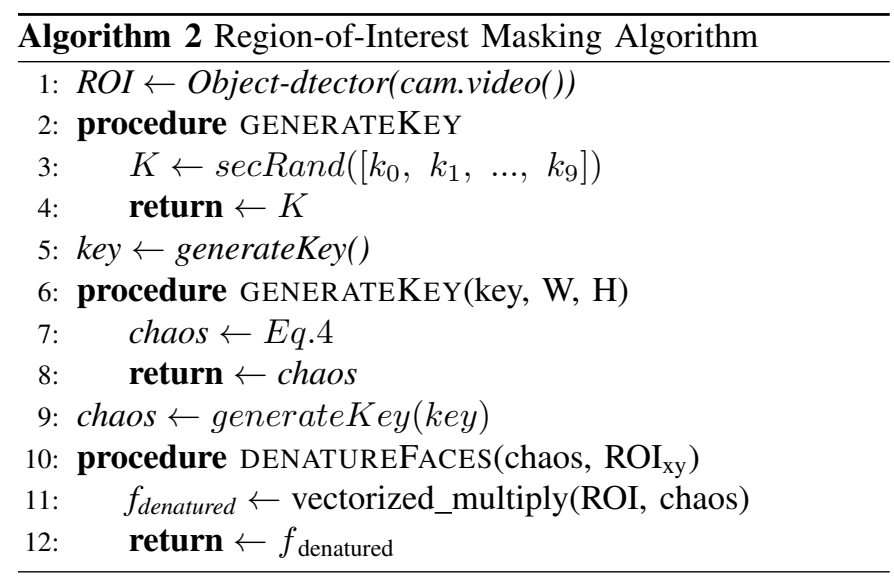

Algorithm 2 shows that a vectorized multiplication is employed. It does contribute to the security of the algorithm. However, it is only suitable for anonymizing small regions on a frame. In our work, we employed it only for denaturing a specific ROI, like faces, which usually is a small portion of a whole frame. However, such an approach is not quite ideal for full frame scrambling. In scenarios where there is a need to ensure E2E privacy for the entire frame, this is not the way to go because multiplication normally requires more time (it takes more gates) than other simpler but more efficient operators like bitwise XOR.

\section{Sinusoidal Chaotic Map (SCM)}

Inspired by the the logistic map [7], [22], [28], a well-known low-dimension chaotic system, a novel lightweight sinusoidal chaotic map (SCM) is proposed for video frame encryption at the edge of the network. As stated in Eq. (5), $\alpha, \beta$ and $\gamma$ are the control parameters, and $x_{0}$ is the initial condition. The SCM system produces a double curve when plotted in an $(x, y)$-plane unlike the simple logistic map, which has a single downward parabolic curve.

$$
x_{n+1}=\frac{\alpha}{2}\left[2 \sin \left(\beta x_{n}\right)+\gamma\left(1-\cos \left(2 \beta x_{n}\right)\right)\right]
$$

After a thorough analysis, a multiplying constant was added to the system to further improve the security of the chaotic sequence generator. It generates a secure and evenly distributed chaotic image. Eq. (6) describes how the key and chaos are generated, with three more parameters added to those stated in Eq. (5). They are a multiplier of value 1624, a scaling parameter $\theta$ and an updating parameter $\delta$.

$$
\begin{aligned}
\text { Key } & =\left[x_{0}, \alpha, \beta, \gamma, \delta, \theta\right] \\
x_{n} & =x_{0} \\
t m p_{1} & =\alpha \times \sin \left(\beta \times x_{n}\right) \\
t m p_{2} & =0.5 \times \alpha \gamma \cos \left(2 \beta \times x_{n}\right) \\
\text { const } & =0.5 \alpha \gamma \\
x_{n+1} & =1624\left[\text { tmp } p_{1}-t m p_{2}+\text { const }\right] \% 1 \\
\text { Chaos } & =\text { append }\left(x_{n+1} \times \theta\right) \\
x_{n} & =\delta \times x_{n+1}
\end{aligned}
$$

The video frame scrambling using the sinusoidal chaotic sequence is performed color-channel-wise simultaneously to reduce the processing time. The image scrambling process comprises a key-generator and chaos-generator modules. The key generator module (key_gen()) generates a key comprising six elements, each 64-bit long, as stated in Eq. (7).

$$
\begin{aligned}
K e y & =g e n \_k e y() \\
& =\left[x_{0}, \alpha, \beta, \gamma, \delta, \theta\right]
\end{aligned}
$$

where $x_{0}$ is the initial condition of the system that triggers the chaotic generator to recursively generate the required set of chaotic pixels. Then, the respective keys for the enciphering of the three color channels of the image are generated by using Eqs. (8) and (9).

$$
\begin{gathered}
K e y_{R}=\left[x_{0}, \alpha_{r}, \beta_{r}, \gamma_{r}, \delta_{r}, \theta_{r}\right]=g e n \_k e y() \\
K e y_{G}=\left[x_{0}, \alpha_{g}, \beta_{g}, \gamma_{g}, \delta_{g}, \theta_{g}\right]=g e n \_k e y() \\
K e y_{B}=\left[x_{0}, \alpha_{b}, \beta_{b}, \gamma_{b}, \delta_{b}, \theta_{b}\right]=g e n \_k e y() \\
K e y_{r}, K e y_{g}, K e y_{b},=g e n \_k e y(), g e n \_k e y(), g e n \_k e y() \\
r_{0}, g_{0}, b_{0}=K e y_{r}[0], K e y_{g}[0], K e y_{b}[0]
\end{gathered}
$$

where $r_{0}, g_{0}$, and $b_{0}$ are the initial values for channels R, G, and B, respectively. Every key element is a 64bit floating point value randomly picked from a domain in the range $(a, b)$, where there are infinitely many real-valued numbers between $a$ and $b$. To enable parallel encryption of the color channels of every frame, the required chaotic images $\left(\right.$ Chaos $_{r}$, Chaos $_{g}$, and Chaos $)$ are produced by using Eqs. (10-12) in parallel.

$$
\begin{aligned}
t_{r} & =\left(1+\text { Key }_{r}[3] \times \sin \left(\operatorname{Key}_{r}[2] \times r_{0}\right)\right) \\
R_{c} & =1624 \times \text {Key }_{r}[1] \times \sin \left(\text {Key }_{r}[2] \times r_{0}\right) \times t_{r} \% 1 \\
r_{0} & =R_{c} \times \text { Key }_{r}[4] \\
\text { Chaos }_{r} & =R_{c} \times \text { Key }_{r}[5]
\end{aligned}
$$




$$
\begin{aligned}
& t_{g}=\left(1+K e y_{g}[3] \times \sin \left(K e y_{g}[2] \times g_{0}\right)\right) \\
& G_{c}=1624 \times K e y_{g}[1] \times \sin \left(K_{e y_{g}}[2] \times g_{0}\right) \times t_{g} \% 1 \\
& g_{0}=G_{c} \times \operatorname{Key}_{g}[4] \\
& \text { Chaos }_{g}=G_{c} \times \text { Key }_{g}[5] \\
& t_{b}=\left(1+\operatorname{Key}_{b}[3] \times \sin \left(\operatorname{Key}_{b}[2] \times b_{0}\right)\right) \\
& \left.B_{c}=1624 \times \operatorname{Key}_{b}[1] \sin \left(\operatorname{Key}_{b}[2] \times b_{0}\right) \times t_{b}\right) \% 1 \\
& b_{0}=B_{c} \times K e y_{b}[4] \\
& \text { Chaos }_{b}=B_{c} \times \operatorname{Key}_{b}[5]
\end{aligned}
$$

Algorithm 3 describes the procedures performed by the privacy-preserving SCM scheme. Frames deemed important are forwarded to remote server, operation centers, and storage sites in scrambled form. The channel keys, chaos, and scrambling processes are performed in parallel.

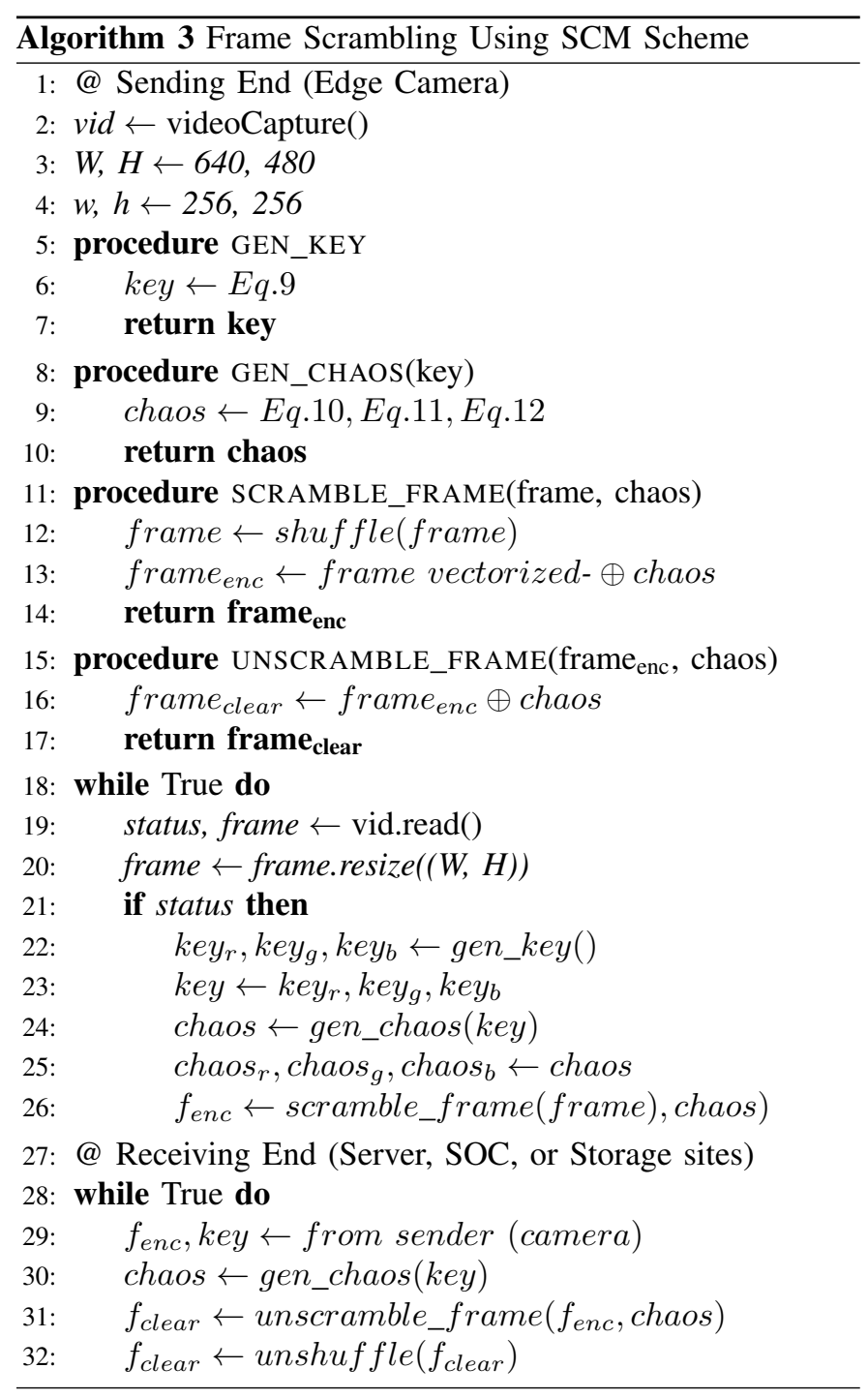

\section{Frame Shuffling to Enhance Security}

To further enhance the security of the proposed schemes, we have introduced a simple but efficient frame shuffling

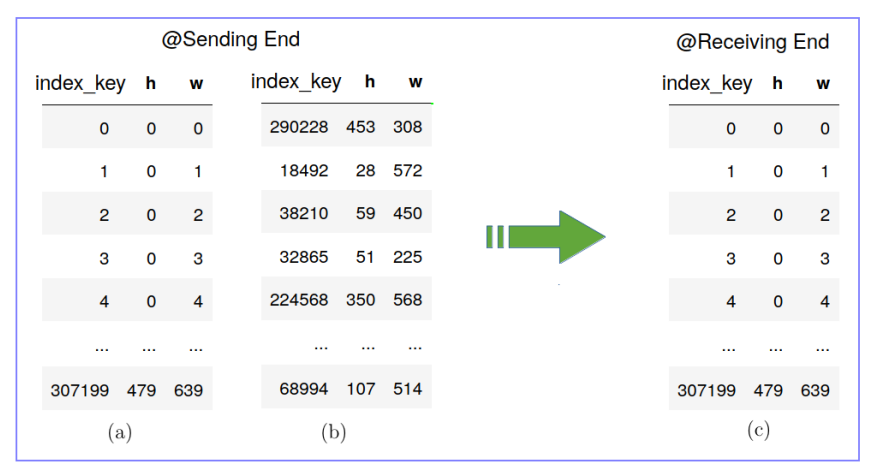

Fig. 6. Frame Shuffling and Unshuffling: (a) Original positions of blocks and (b) shuffled positions of blocks at sending end, and (c) restored positions of blocks at receiving end.

algorithm. It randomizes the pixels of a frame in blocks of sizes $1 \times 1,4 \times 4,8 \times 8,16 \times 16$, or $32 \times 32$. This increases the diffusion of pixels or block of pixels which increases clearframe sensitivity important for cutting down on the probability of differential attacks. The procedure is depicted in Algorithm 4 pythonically.

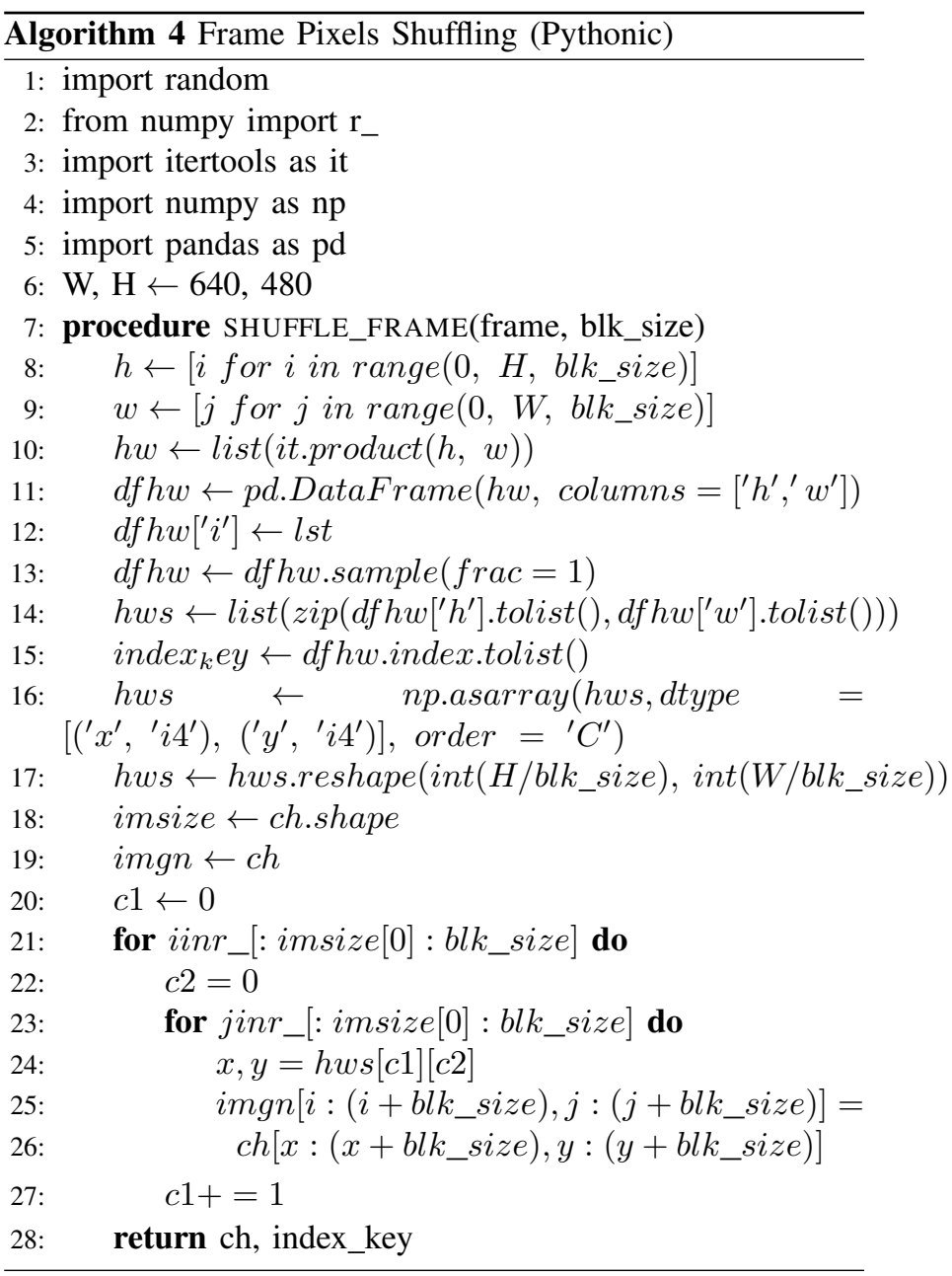

As depicted in Algorithm 4, all possible block $(x, y)$ positions are combined together as tuples in a list. Then, they are truly shuffled using a data structure or data frame after an index has been added. The shuffling method employed is 
inherently irreversible; however, the index is used to reproduce the original tuple positions. The block size, and index_key employed to shuffle the input frame at the sending end must be securely forwarded to the receiving end. At the receiving end, the original positions of the blocks in the frame are restored by simply sorting the index_key column. Figure 6(a) shows the original positions of blocks in an input frame, and (b) portrays the randomized positions $((x, y) \leftarrow(h, w))$ of the blocks along with their respective index_key values at the sending end. At the receiving end, Fig. 6(c) shows the restored positions of blocks in the received frame.

\section{EXPERIMENTS AND RESULT ANALYSIS}

In the experimental setup, smart CCTV cameras deployed at the edge of the network with a Raspberry Pi 4 incorporated into them, whose specifications are provided in Table II, are employed. These low-cost, tiny single board computers (SBC) are vital for enforcing the privacy-preserving mechanisms on the video frames at the point of video frame creation to ensure E2E privacy protection. The implementation is done using Python 3.7.4 with multithreading and multiprocessing enabled. Besides, the video frame employed for the experimental analysis and implementation has a size of $480 \mathrm{P}(480 \times 640 \times 3)$ and three color channels (RGB) with a pixel depth of 8 bits.

In addition to performance in terms of number of frames the proposed schemes can process per second, a comprehensive experimental study has been conducted, including a comparative study of 2D-ReC, DyCIE, and SCM with two classic solutions, RC4 and AES, plus two recently reported efforts.

TABLE II

SPECIFICATION OF THE RASPBERRY PI 4

\begin{tabular}{|l|l|}
\hline Parameters & Specifications \\
\hline \hline CPU type/speed & $\begin{array}{l}\text { Quad core Cortex-A72 (ARM v8) 64-bit SoC } \\
\text { @ } 1.5 \mathrm{GHz}\end{array}$ \\
\hline RAM size & $4 \mathrm{~GB} \mathrm{LPDDR} 4-2400$ SDRAM \\
\hline Integrated Wi-Fi & $2.4 \mathrm{GHz}$ and $5 \mathrm{GHz}$ \\
\hline Ethernet speed & $1 \mathrm{Gbps}$ \\
\hline Camera port & 2 -lane MIPI CSI \\
\hline Bluetooth & 5.0 \\
\hline Power Requirement & $3 \mathrm{~A}, 5 \mathrm{~V}$ \\
\hline Operating System & Debian Linux 10 based \\
\hline
\end{tabular}

\section{A. Dynamic Chaotic Image Enciphering Scheme (DyCIE)}

The speed analysis refers to the amount of time consumed for scrambling a frame or image. It includes time required for preliminary processing, splitting the input frame into the three colors, encrypting each channel in parallel, and stacking them up together. On average, the proposed DyCIE scheme can scramble about 10.274 fps.

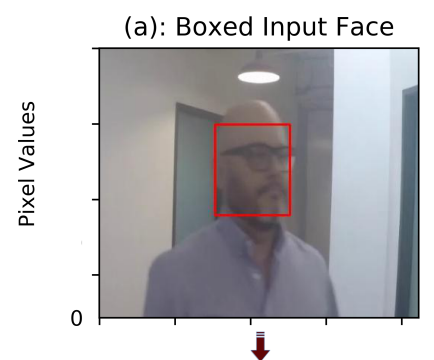

(c): Histogram of (a)

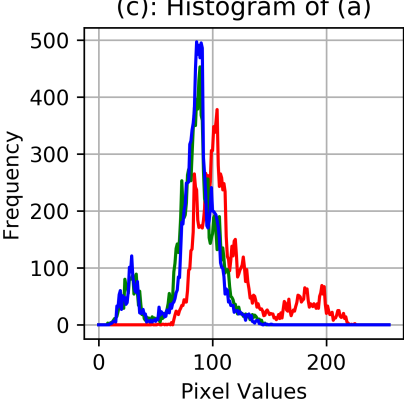

(b): Enciphered Face

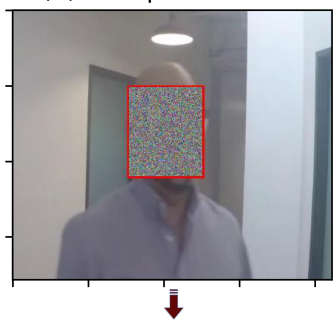

(d): Histogram of (b)

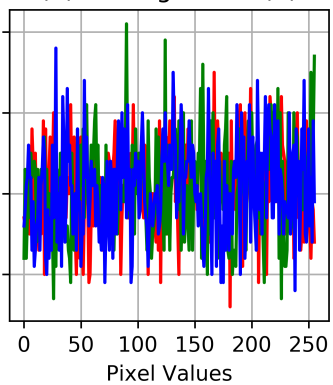

Fig. 7. Histogram: (a) plain face, (b) cipher of face (a), (c) histogram of clear face, and (d) randomized histogram of cipher face (b).

\section{B. RoI-Mask For Attributes Denaturing}

Figure 7 demonstrates a sample histogram analysis for our RoI-Mask scheme. Figure 7(a) is a clear boxed input face and its ciphered version is shown in Fig. 7(b). Figure 7(c) shows that the frequency distribution of the clear input image in Fig. 7(a) is not uniform. In contrast, the frequency distribution of the scrambled face in Fig. 7(b) has become uniform as portrayed in Fig. 7(d). This validates that the privacyprotection scheme is secure against a frequency analysis attack. Besides, it is computationally efficient for smaller ROIs and secure in that it passes all other security tests. It is also proved to be robust against the possible statistical and computational attacks. All the tests carried out are depicted in Table IV.

\section{Impacts of Block Shuffling}

Figure 8 presents the experimental results of the frame shuffling process. Figure 8(a) shows the clear input frame. Then, before the actual scrambling process, the input frame is shuffled in blocks of size $32 \times 32$ as depicted in Fig. 8(b). Figure 8(c) shows a frame shuffled with blocks of size 16 . Figures $8(d),(e)$, and (f) show the outputs of shuffling with blocks of size $8 \times 8,4 \times 4$, and $1 \times 1$, respectively. In all of them, there is nothing recognizable. But the degree of pixelation decreases and the degree of confusion increases as the block size decreases. For computational efficacy, we employed a block size of $32 \times 32$ in this work.

\section{Sinusoidal Chaotic Maps (SCM) Scheme}

A thorough experimental study was conducted on the novel SCM scheme with detailed analyses based on standard computational and statistical security parameters. Then, the results have clearly validated its security and computational efficacy. 


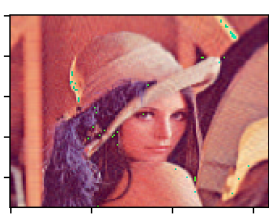

(a)

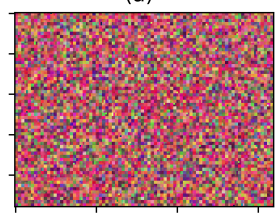

(d)

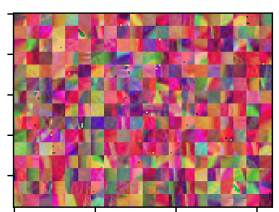

(b)

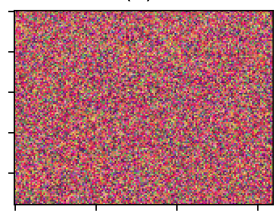

(e)

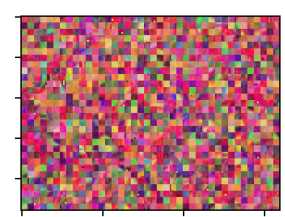

(c)

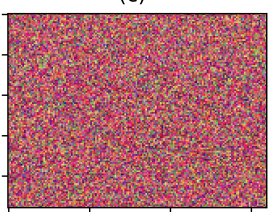

(f)
Fig. 8. Frame shuffling with different block sizes: (a) Clear input frame, (b) shuffled with block_size: $32 \times 32$, (c) shuffled with block_size:16 $\times 16$, (b) shuffled with block_size: $8 \times 8$, (b) shuffled with block_size: $4 \times 4$, (b) shuffled with block_size: $1 \times 1$

For a comprehensive performance and security analysis of the novel SCM scrambling scheme, several cases and computational-and-statistical security parameters have been considered. The properties, computational and statistical security parameters considered include functional tests, Lyapunov Exponents, time complexity, visual assessment, key space and chaos size analysis, key sensitivity analysis, statistical analysis, Peak Signal to Noise Ratio (PSNR), Number of Pixels Change Rate (NPCR), Unified Average Changing Intensity (UACI), histogram analysis, correlation analysis, differential analysis, and Information Entropy analysis.

\section{Functional Test}

As portrayed in Fig. 9, the input frames (a) and (c) are successfully scrambled into a totally random version using the SCM method proving its functionality. The inverse process of scrambling also successfully performed.

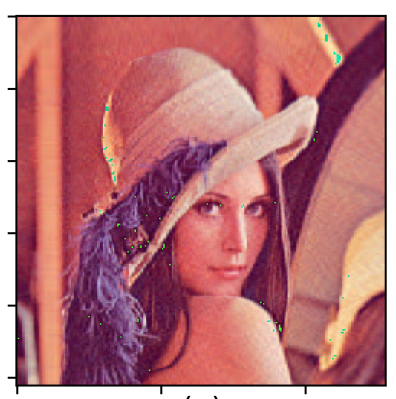

(a)

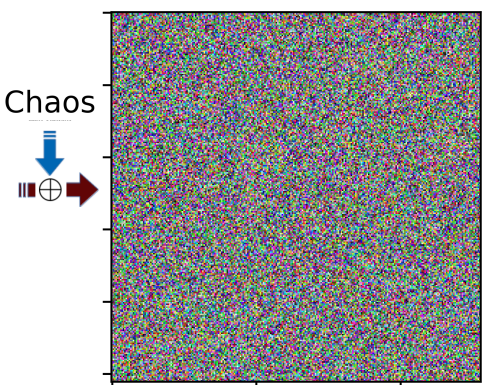

(b)
Fig. 9. Frame scrambling:(a) is a clear image; whereas (b) is its cipher image.

2. Lyapunov Exponents Analysis The Lyapunov exponents (LE) measure the predictability and sensitivity of a system to changes in its initial conditions, often termed as stability. They can be considered as the average logarithmic rate of separation or convergence of two nearby points of two time series $X_{n}$ and $X_{n+1}$, separated by an initial distance computed by using Eq. (13). LE indicates how evenly the points of a chaotic sequence generator are distributed. It is computed by using Eq. (14).

$$
\Delta X=\left\|X_{n+1}-X_{n}\right\|^{2}
$$

$$
L E=\lim _{N \rightarrow+\infty} \frac{1}{N} \sum_{n=1}^{N} \log \left|\frac{\partial x_{n+1}}{\partial x_{n}}\right|
$$

The Lyapunov exponents of SCM method is computed as follows based on Eq. (14).

$$
\frac{d x_{n+1}}{d x_{n}}=\frac{d\left[\alpha \times \sin \left(\beta x_{n}\right)-t m p \times \cos \left(2 \beta x_{n}\right)+t m p\right]}{d x_{n}}
$$

where tmp $=0.5 \times \alpha \times \gamma$. Then, the derivatives on Eq. (15) were performed following calculus rules like product rule $\left((f(x) g(x))^{\prime}=f^{\prime}(x) g^{\prime}(x)\right)$ and chain rule $\left(\left(f(g(x))^{\prime}=\right.\right.$ $\left.(f \circ g)^{\prime}=f^{\prime}(g(x)) * g^{\prime}(x)\right)$ that resulted in Eq. (16)

$$
\frac{d x_{n+1}}{d x_{n}}=\alpha \beta \cos \left(\beta x_{n}\right)+\alpha \beta \gamma \sin \left(2 \beta x_{n}\right)
$$

By substituting $\frac{d x_{n+1}}{d x_{n}}$ in Eq. (14) with its expression given by Eq. (16), the final formula for computing the Lyapunov exponents of our SCM scheme is obtained as Eq. (17). Figure 10 presents the Lyapunov Diagram of the proposed Sinusoidal Map generated by using Eq. (17).

$$
L E=\lim _{N \rightarrow+\infty} \frac{1}{N} \sum_{n=1}^{N} \log \left|\alpha \beta\left[t m p_{1}+t m p_{2}\right]\right|
$$

where $t m p_{1}=\cos \left(\beta \times x_{n}\right)$ and $t m p_{2}=\gamma \times \sin \left(2 \beta \times x_{n}\right)$.

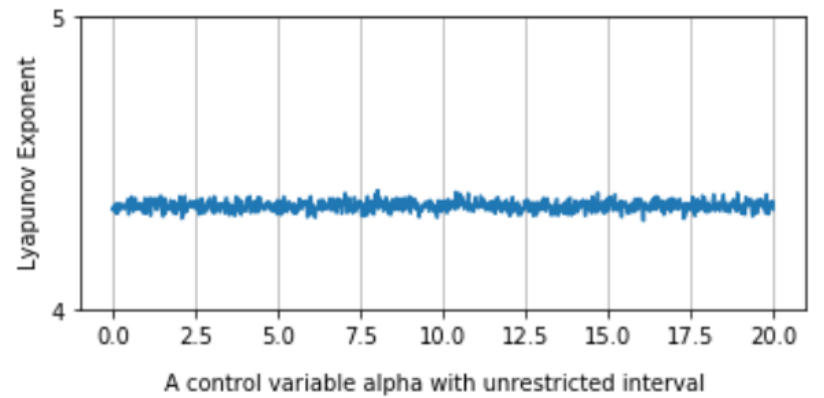

Fig. 10. Lyapunov Diagram of the proposed Sinusoidal Map.

Figure 10 demonstrates that Lyapunov exponents of the sinusoidal map are evenly distributed over unrestricted range. For illustration purpose, the control variable of SCM model is fixed at 20 but it can be extended to any greater value. The Lyapunov exponents vary within 4.36 and 4.45 signifying the uniformity of the proposed SCM scheme. Meanwhile, the popular Logistic map [28] has uneven Lyapunov properties and experiences chaotic properties only within a restricted range of the control variable, between 3.57 and 4 . Besides, the Lyapunov exponents of the logistic map vary between -4 and 1 as depicted in Fig. 11 indicating non-uniformity. Comparing Fig. 10 to Fig. 11 clearly shows that SCM scheme achieves a much more even distribution.

\section{Encryption Time}

Video frames are bulky, and a good-performing scrambling technique is expected to take less time to encrypt a frame. The computational speed is not only affected by the algorithm design but also by other multiple factors, including the type of hardware platform and the programming language employed. In this work, Raspberry PI 4 is used as the edge device. 


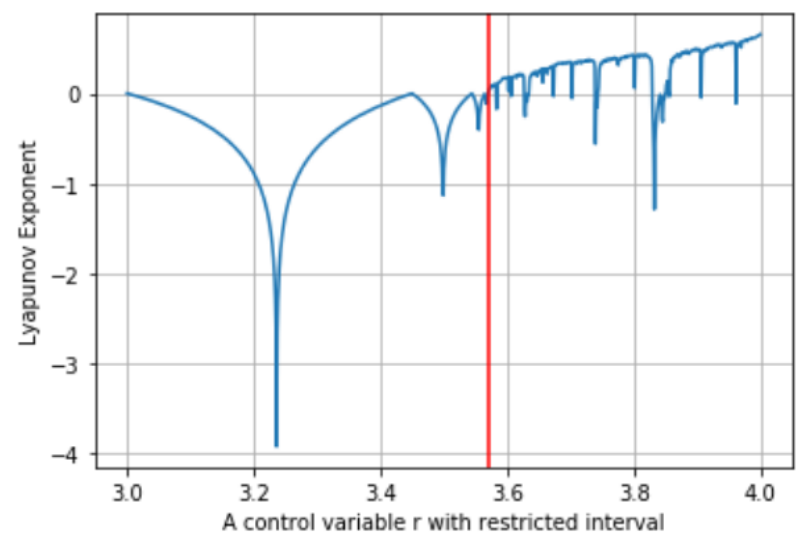

Fig. 11. Lyapunov Diagram of the Logistic Map [28].

Besides, our scheme is implemented using both Python 3.7.4 and $\mathrm{C}++$. As expected, the implementation based on $\mathrm{C}++$ is much faster than the python implementation. However, for the purpose of comparative analysis with equivalent previous works, the python implementation is consistently considered.

Channel keys and chaoses are generated in parallel. Besides, the scrambling processes are performed channel-wise in parallel. The corresponding pixels of the clear frame and chaos are mixed in parallel using vectorized xor. This approach speeds up the scrambling processes. Our SCM scheme, therefore, can scramble 10.469 frames per second (fps); that is, it takes 95.52 milliseconds to encrypt a single frame.

\section{Visual Assessment}

A scrambling scheme is said to be good if there is no recognizable visual information on the cipher image. As illustrated in Fig. 9, the scrambled frame, (b), contain no visually recognizable information about the clear frame, (a). The encrypted image is random and highly disordered proving that the SCM scheme is resistant against any visual assessment attack. This test was conducted on multiple frames.

\section{Key Space Analysis}

In the common practice of cryptography, the security of a scheme depends on the length of the keys. It should be sufficiently large and unbreakable by brute force analysis in a reasonably short period of time. Our SCM scheme has a key that comprises six parameters, each with a 64-bit long floating-point decimal value. Hence, the total key space of SCM scheme is $2^{384}$, which is sufficiently large to resist any possible exhaustive key search analysis attack. Actually, it is much longer than the lower boundary of a secure key space in the practice of symmetrical cryptography, $2^{128}$.

\section{Histogram Analysis}

Histogram is the frequency description of each pixel value of a frame. The histograms of the scrambled frames must be totally statistically different from that of corresponding original images. Besides, the scheme is said to be resistant against statistical histogram attacks if it has uniform histograms.

Figure 12 shows the histograms of the unscrambled frame channels and their corresponding scrambled versions. The histograms of the scrambled versions shown in Fig. 12 column
4, unlike that of the plain frame channels depicted Fig. 12 column 2, are uniform proving the security of the scheme. This demonstration substantiates the robustness of the scheme against any histogram analysis attack.

\section{Statistics of the chaos}

For an ideally uniformly distributed 8-bit image, the pixel values are expected to be uniformly distributed between 0 and 255, inclusively. Hence, the corresponding ideal reference statistical descriptions are provided in Table III. A robust scrambling scheme is supposed to produce statistical descriptions close to these ideal values. The cipher of SCM scheme has statistical descriptions, provided in the last row of Table III, which almost achieve the ideal mean, standard deviation (std), minimum ( $\mathrm{min}$ ), $25^{\text {th }}$ percentile, $50^{\text {th }}$ percentile, $75^{\text {th }}$ percentile, and maximum (max) values. This again validates the uniformity of SCM scheme.

TABLE III

STATISTICAL DESCRIPTIONS

\begin{tabular}{|c|c|c|c|c|c|c|}
\hline mean & std & $\min$ & $25 \%$ & $50 \%$ & $75 \%$ & $\max$ \\
\hline \multicolumn{7}{|c|}{ Ideal Statistical Description } \\
\hline 127.5 & 73.9 & 0 & 63.75 & 127.5 & 191.25 & 255 \\
\hline \multicolumn{7}{|c|}{ Statistical Descriptions of The Proposed Scheme } \\
\hline 127.98 & 73.55 & 0 & 64 & 128 & 192 & 255 \\
\hline
\end{tabular}

\section{Key Sensitivity Analysis}

Key sensitivity analysis measures the difference between two ciphers obtained by enciphering the same plain frame using two slightly different keys. Precisely, a pair of keys that differ only by one bit must produce two entirely different ciphers. The difference between the two ciphers is measured by calculating the NPCR and UACI of the ciphers. The rule of thumb is that these two ciphers must achieve NPCR $>99 \%$ and $U A C I$ around $33 \%$ in order for the scrambling scheme to be resistant against differential attacks.

Defined by Eq. (18), UACI is employed to measure the average intensity difference in a color channel between its two cipher versions $C_{1}(i, j)$ and $C_{2}(i, j)$.

$$
U A C I=\frac{1}{H * W}\left[\sum_{i, j} \frac{C_{1}(i, j)-C_{2}(i, j)}{255}\right] * 100 \%
$$

The NPCR measures the change rate of the number of pixels of the cipher-frame when only a bit of the original key or pixel is modified. It is defined by Eq. (19).

$$
N P C R=\frac{\sum_{i, j} D(i, j)}{H * W} * 100 \%
$$

where $H$ and $W$ are the height and width of the cipher images, encrypted using $k e y_{1}$ and $k e y_{2}$ that vary from each other by only a bit. $D(i, j)$ is defined by Eq. (20).

$$
D(i, j)= \begin{cases}1, & \text { if } C_{1}(i, j) \neq C_{2}(i, j) \\ 0, & \text { else }\end{cases}
$$

The proposed SCM scheme achieves a $U A C I=33.5 \%$ and an NPCR $=99.69 \%$, which means our proposed video frame scrambling scheme meets the key sensitivity requirements and 
(a): RGB Input Image

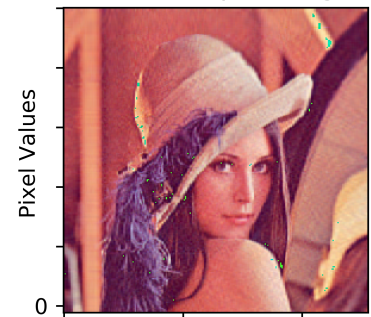

(a1): plain R-Channel of (a)

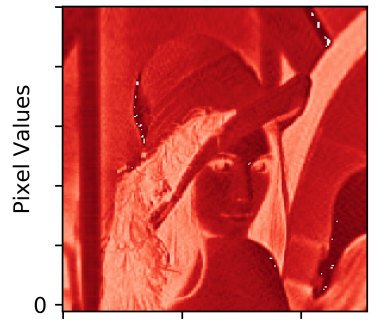

(a2): plain G-Channel of (a)

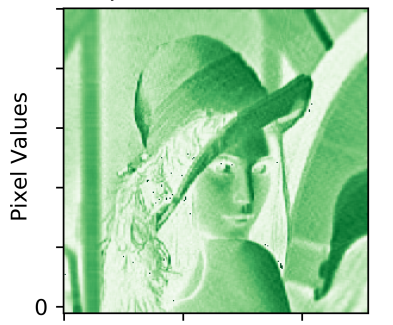

(a3): plain B-Channel of (a)

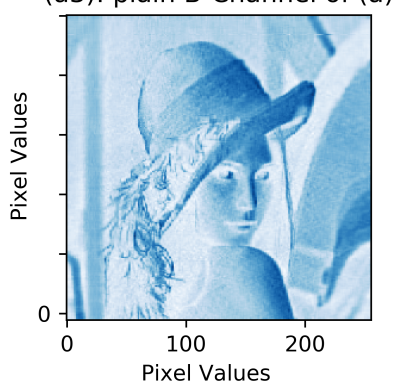

Histogram of (a)

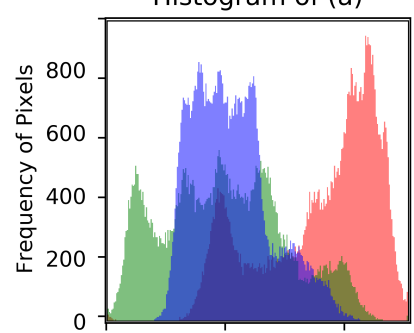

Histogram of clear R

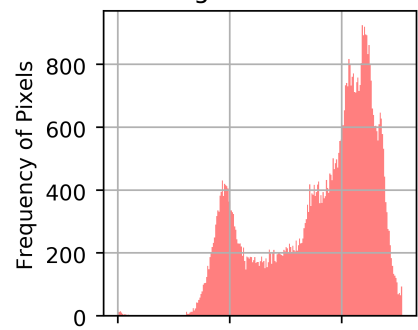

Histogram of clear R
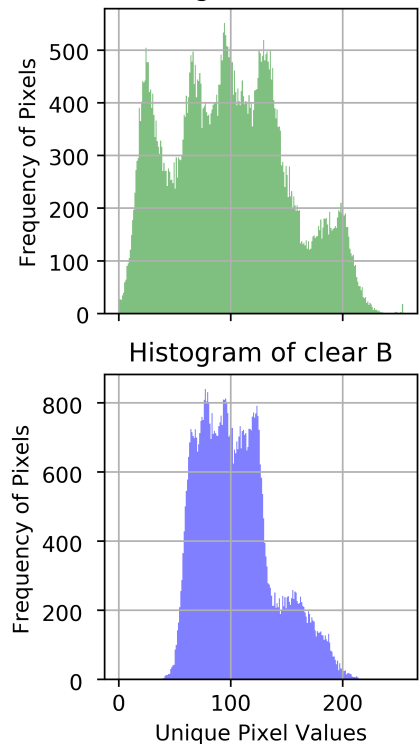

(b): Cipher of (a)

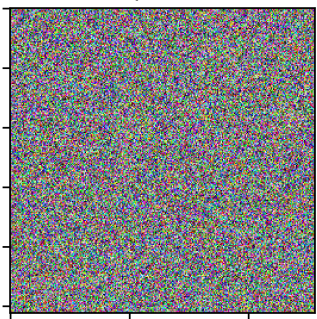

(b1): Cipher of R

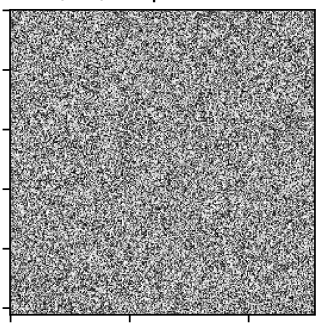

(b2): Cipher of G

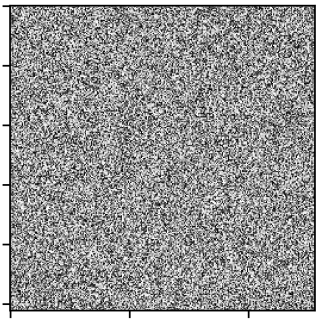

(b3): Cipher of B

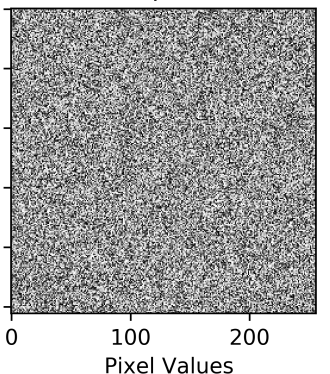

Histogram of (b)

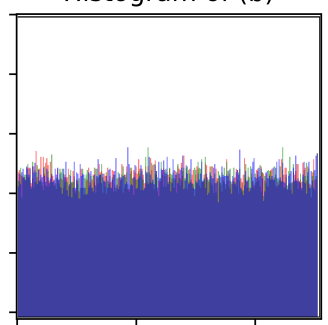

Histogram of R in (b1)

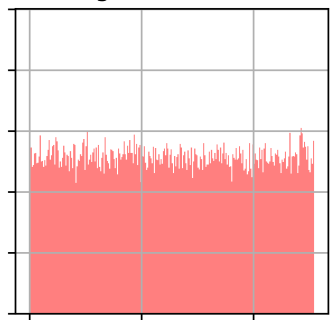

Histogram of G in (b2)

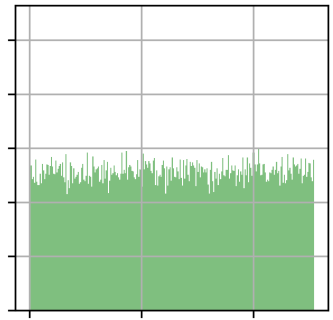

Histogram of G in (b2)

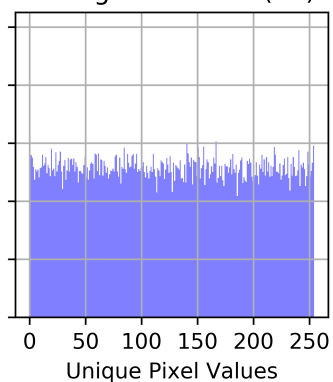

Fig. 12. Histogram Analysis: Column 1 comprises the clear input frame and its color channels R, G, and B from top to bottom. Column 2 represents the histograms of the clear input frame and its R, G, and B channels in the order from top to bottom. Column 3 comprises the ciphers of the input frame and its color channels in column 1 in the same order. Column 4 illustrates the histograms of the cipher frame and its channels in column 3.

it is secure against possible differential analysis attack. This is also referred to as the differential analysis.

\section{Clear-frame Pixel Sensitivity}

Similar to the measure of key sensitivity, the clear-image sensitivity states that a given plain original image and another version with a single bit change should produce completely different ciphers when encrypted using the same key. The NPCR and UACI are again employed to compute the difference between the ciphers. Then, the scheme that produce an NPCR over $99 \%$ and a UACI over $33 \%$ is considered to be highly sensitive to any pixel change and secure against differential attack. Our SCM scheme achieves an NPCR of $99.23 \%$ and a UACI of $33.12 \%$.

\section{Peak Signal to Noise Ratio (PSNR) Analysis}

PSNR helps us measure the difference between the original signal, a frame in our case, and a noise, in our case the enciphered frame. It is defined as the base-10 logarithm of the ratio of the square of the maximum pixel value to the mean square error (MSE) of the plain and enciphered frames.

Given a plain $W \times H$ 2D color component of an RGB frame, $I$, and its noisy/scrambled version $C$, the $P S N R$ is defined as ensues by Eq. (21):

$$
\begin{aligned}
P S R N & =10 * \log _{10}\left(\frac{M A X_{I}^{2}}{M S E}\right) \\
M S E & =\frac{1}{W * H} \sum_{i=0}^{W-1} \sum_{j=0}^{H-1}[I(i, j)-C(i, j)]^{2}
\end{aligned}
$$

Here, $M A X_{I}$ is the maximum possible pixel value of the plain channel image. When the pixels are represented using eight bits per sample, this is 255. The PSNR of a robust scrambling scheme is expected to be low because the MSE of the plain and scrambled images is expected to be higher. 
The average PSNR of the SCM scheme is $7.834 d B$, which is a really great result that signifies the complete difference between the plain and cipher frames.

\section{Information Entropy Analysis}

The information entropy, $H(C)$, defined by Eq.(22), measures how randomly the $N$ pixels of the scrambled image $C$ are shuffled. For an 8-bit pixel representation, the ideal value of the entropy is $H(C)=8$. Hence, for an 8-bit pixel representation, a secure scrambling scheme is supposed to have an information entropy value very close to 8.0.

$$
H(C)=-\sum_{i=0}^{N-1} P\left(C_{i}\right) \log _{2}\left(C_{i}\right)
$$

SCM scheme produces entropy values of 7.999 , very close to the ideal value, which proves its security against entropy analysis attack.

\section{Correlation Analysis}

Video frames are so bulky characterized by very strong redundancy and correlations amongst adjacent pixels. Hence, to make correlation analysis attack irrelevant, a robust scrambling scheme should be able to diffuse and shuffle frames to produce a cipher frame with uncorrelated adjacent pixels. The correlation parameter computes the correlation among the adjacent pixels of the cipher frame. A secure framescrambling scheme is required to produce a cipher frame with uncorrelated or nearly zero correlation between adjacent pixels. The correlation analysis in the horizontal, vertical and diagonal directions is performed between pairs of clear-frame channels and cipher-frame channels by using Eq. (23).

$$
\begin{array}{r}
r_{x y}=\frac{\operatorname{cov}(x, y)}{\sqrt{D(x) D(y)}} \\
\operatorname{cov}(x, y)=\frac{1}{N} \sum_{i=1}^{N}\left(x_{i}-E\left(x_{i}\right)\right)\left(y_{i}-E\left(y_{i}\right)\right) \\
E(x)=\frac{1}{N} \sum_{i=1}^{N} x_{i} \\
D(x)=\frac{1}{N} \sum_{i=1}^{N}\left(x_{i}-E(x)\right)^{2}
\end{array}
$$

Our experimental analysis on SCM scheme shows that the correlations of the cipher pixels in the horizontal, vertical, and diagonal directions are nearly zero (0.00007, 0.00006, and 0.00029 , respectively). These numbers corroborate the robustness and security of SCM scheme against correlation analysis attacks. More illustratively, Figs. 13 (ah), (av), and (ad) portray the horizontal, vertical, and diagonal correlations of the adjacent pixels of the clear input image in Fig. 13(a), respectively. On the other hand, Figs. 13 (bh), (bv), and (bd) illustrate the randomized horizontal, vertical, and diagonal correlations of the adjacent pixels of the cipher in Fig. 13(b) proving its robustness against correlation attacks.

\section{E. Comparative Analysis}

In addition to a comprehensive security and performance analysis, we have conducted a comparative study not only among our proposals, but also with contemporary chaotic schemes and most widely used data encryption schemes. Our proposed chaotic schemes compared here include 2D-ReC, DyCIE, and SCM. Four benchmarks were selected based on a combination of performance speed, being state-of-theart, security, and the basic technique employed. They are RC4, AES, an image encryption scheme based on simple logistic chaotic map proposed by Liu et al [21], and an image encryption scheme based on double spiral scans and chaotic maps proposed by Tang et al [33]. RC4 is one of the fastest stream ciphers; it has some key security issues, though. AES is the most widely used encryption scheme in today's Internet TLS/SSL. Works introduced by Liu et al and Tang et al are chaotic-based encryption schemes. The Liu's approach is a relatively lighter scheme designed based on a parameter-varied non-linear logistic chaotic map, the simplest and well-studied chaotic map. It offers good speed and security unlike other higher dimension chaotic systems. The Tang's proposal is one of the most recently published chaotic encryption schemes. It is a secure scheme designed based on low complexity double spiral scans and a chaotic map.

Table IV summarizes the results of the comparison study. Schemes proposed in this paper have achieved very good security and performance. Our schemes were carefully designed to break any pixel adjacency; as a result, they have slightly better score in terms of the correlation parameter. Besides, one of the key design goal of this work is making the schemes lightweight. Hence, our schemes stand out in terms of speed. They have way much better frame processing speed than the benchmark methods. 2D-ReC, DyCIE, and SCM can process about $5 \mathrm{fps}, 10.2 \mathrm{fps}$, and $10 \mathrm{fps}$, respectively.

Overall, the SCM frame enciphering scheme is superior to the other schemes in terms of security and the range of parameter values. It is more robust than the other methods when measured in terms of standard computational and statistical security parameters. It can optimally run on edge cameras where there is resource constraint. The DyCIE scheme works well in a very resource-constrained environment. But $2 \mathrm{D}-\mathrm{ReC}$ is much slower. RoI-mask is not compared with the others because it is not for full-frame protection, instead, it is specially designed for protecting the small regions of interest. RoI-Mask employs a vectorized multiplication operator for mixing frame and chaotic pixels in parallel. It is capable of processing a $480 \mathrm{P}$ frame every 49 milliseconds.

\section{COnClusions}

Privacy is fundamental to healthy operation of surveillance systems; as a result, it has been among the major concerns while a bunch of smart cities heavily rely on surveillance systems. In this work, we proposed three slenderized mechanisms for E2E privacy-preserving in VSS. The design rationales and principles are discussed in detail. A comprehensive security and experimental analysis corroborates that the proposed schemes are secure and faster than existing popular encryption methods when used for video frames encryption at the edge of the network. The technology for privacy-aware and privacy-preserving smart cities is far from 
(a): plain R-Channel

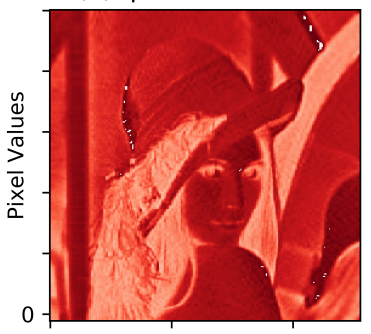

(b): Cipher of R-Channel

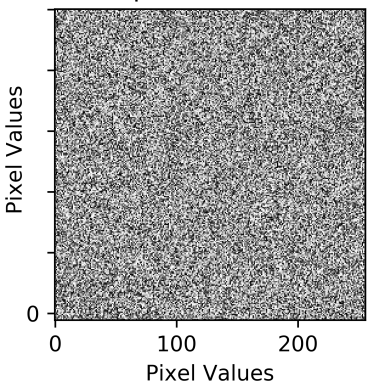

(ah)

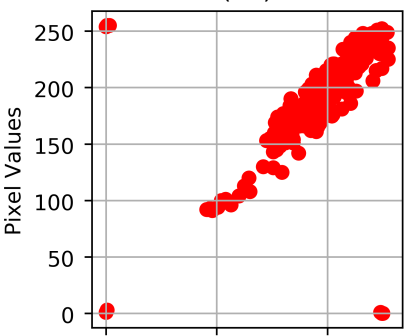

(bh)

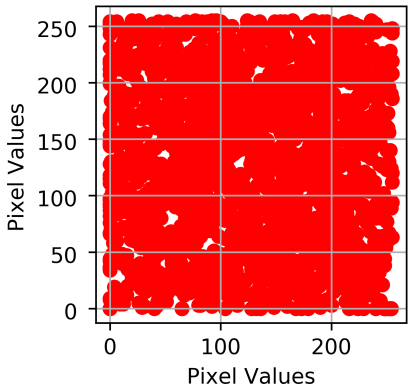

(av)

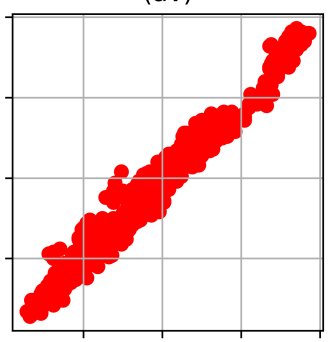

(bv)

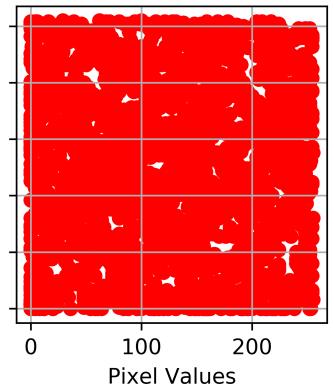

(ad)

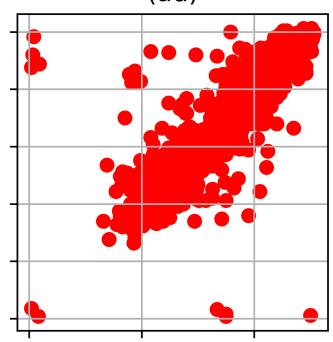

(bd)

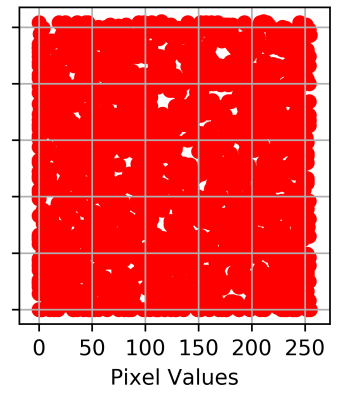

Fig. 13. An Illustration of Scatter plots of the horizontal, vertical, and diagonal correlations of the clear R-channel in (a) and its cipher frame in (b).

mature, a lot of open questions yet to be tackled. We hope this effort will inspire more discussions and novel solutions from the edge computing and smart cities community.

\section{REFERENCES}

[1] A. Artusi, R. K. Mantiuk, T. Richter, P. Hanhart, P. Korshunov, M. Agostinelli, A. Ten, and T. Ebrahimi, "Overview and evaluation of the jpeg xt hdr image compression standard," Journal of Real-Time Image Processing, vol. 16, no. 2, pp. 413-428, 2019.

[2] M. Asim and V. Jeoti, "On image encryption: Comparison between aes and a novel chaotic encryption scheme," in 2007 International Conference on Signal Processing, Communications and Networking. IEEE, 2007, pp. 65-69.

[3] A. Cavallaro, "Privacy in video surveillance [in the spotlight]," IEEE Signal Processing Magazine, vol. 2, no. 24, pp. 168-166, 2007.

[4] N. Chen and Y. Chen, "Smart city surveillance at the network edge in the era of iot: opportunities and challenges," Smart Cities, pp. 153-176, 2018.

[5] N. Chen, Y. Chen, X. Ye, H. Ling, S. Song, and C.-T. Huang, "Smart city surveillance in fog computing," in Advances in mobile cloud computing and big data in the 5G era. Springer, 2017, pp. 203-226.

[6] J. Clement, "Global digital population as of july 2020," https://www.statista.com/statistics/617136/digital-populationworldwide/, 2020 (accessed on August 24, 2020).

[7] M. J. Feigenbaum, "The onset spectrum of turbulence," Physics Letters A, vol. 74, no. 6, pp. 375-378, 1979.

[8] A. Fitwi and Y. Chen, "Privacy-preserving selective video surveillance," in 2020 29th International Conference on Computer Communications and Networks (ICCCN). IEEE, 2020, pp. 1-10.

[9] A. Fitwi, Y. Chen, and S. Zhu, "Prise: Slenderized privacy-preserving surveillance as an edge service," in 2020 IEEE 6th International Conference on Collaboration and Internet Computing $(\mathrm{CIC})$. IEEE, 2020, pp. 125-134.

[10] A. Fitwi, Y. Chen, S. Zhu, E. Blasch, and G. Chen, "Privacy-preserving surveillance as an edge service based on lightweight video protection schemes using face de-identification and window masking," Electronics, vol. 10, no. 3, p. 236, 2021.

[11] A. Fitwi, M. Yuan, S. Y. Nikouei, and Y. Chen, "Minor privacy protection by real-time children identification and face scrambling at the edge," EAI Endorsed Transactions on Security and Safety, vol. 18, no. 3, 2020.

[12] A. H. Fitwi, D. Nagothu, Y. Chen, and E. Blasch, "A distributed agentbased framework for a constellation of drones in a military operation," in 2019 Winter Simulation Conference (WSC). IEEE, 2019, pp. 25482559 .
[13] A. H. Fitwi and S. Nouh, "Performance analysis of chaotic encryption using a shared image as a key," Zede Journal, vol. 28, pp. 17-29, 2011.

[14] J. Gleick, Chaos: Making a new science. Open Road Media, 2011.

[15] B. Harris and R. Hunt, "Tcp/ip security threats and attack methods," Computer communications, vol. 22, no. 10, pp. 885-897, 1999.

[16] P. Korshunov and T. Ebrahimi, "Using face morphing to protect privacy," in 2013 10th IEEE International Conference on Advanced Video and Signal Based Surveillance. IEEE, 2013, pp. 208-213.

[17] V. Kumar and J. Svensson, Promoting social change and democracy through information technology. IGI Global, 2015.

[18] D. Levy, "Chaos theory and strategy: Theory, application, and managerial implications," Strategic management journal, vol. 15, no. S2, pp. 167-178, 1994.

[19] R. Li, Q. Liu, and L. Liu, "Novel image encryption algorithm based on improved logistic map," IET Image Processing, vol. 13, no. 1, pp. 125-134, 2018.

[20] L. Lin and N. Purnell, "A world with a billion cameras watching you is just around the corner," The Wall Street Journal, 2019.

[21] L. Liu and S. Miao, "A new image encryption algorithm based on logistic chaotic map with varying parameter," SpringerPlus, vol. 5, no. 1, p. 289, 2016.

[22] R. M. May, "Simple mathematical models with very complicated dynamics," Nature, vol. 261, no. 5560, pp. 459-467, 1976.

[23] E. M. Newton, L. Sweeney, and B. Malin, "Preserving privacy by deidentifying face images," IEEE transactions on Knowledge and Data Engineering, vol. 17, no. 2, pp. 232-243, 2005.

[24] S. Y. Nikouei, R. Xu, Y. Chen, A. Aved, and E. Blasch, "Decentralized smart surveillance through microservices platform," in Sensors and Systems for Space Applications XII, vol. 11017. International Society for Optics and Photonics, 2019, p. 110170K.

[25] S. Y. Nikouei, R. Xu, D. Nagothu, Y. Chen, A. Aved, and E. Blasch, "Real-time index authentication for event-oriented surveillance video query using blockchain," in 2018 IEEE International Smart Cities Conference (ISC2). IEEE, 2018, pp. 1-8.

[26] J. R. Padilla-López, A. A. Chaaraoui, and F. Flórez-Revuelta, "Visual privacy protection methods: A survey," Expert Systems with Applications, vol. 42, no. 9, pp. 4177-4195, 2015.

[27] W. B. Pennebaker and J. L. Mitchell, JPEG: Still image data compression standard. Springer Science \& Business Media, 1992.

[28] S. Phatak and S. S. Rao, "Logistic map: A possible random-number generator," Physical review E, vol. 51, no. 4, p. 3670, 1995.

[29] Q. M. Rajpoot and C. D. Jensen, "Security and privacy in video surveillance: Requirements and challenges," in IFIP International Information Security Conference. Springer, 2014, pp. 169-184.

[30] L. Rakhmawati et al., "Image privacy protection techniques: A survey," in TENCON 2018-2018 IEEE Region 10 Conference. IEEE, 2018, pp. 0076-0080. 
TABLE IV

Comparative SECURITy AND Performance ANALYsis

\begin{tabular}{|c|c|c|c|c|c|c|c|}
\hline Parameter & DyCIE & SCM & 2D-ReC & RC4 & AES & Liu's & Tang's \\
\hline mean & 127.5 & 127.43 & 127.49 & 127.35 & 173.31 & 127.89 & 127.74 \\
\hline std & 73.9 & 73.75 & 73.897 & 73.92 & 73.93 & 73.863 & 73.78 \\
\hline $\min$ & 0 & 0 & 0 & 0 & 0 & 0 & 0 \\
\hline $25 \%$ & 63.75 & 63 & 63 & 63 & 63 & 64 & 63 \\
\hline $50 \%$ & 127.5 & 127 & 127 & 127 & 127 & 128 & 128 \\
\hline $75 \%$ & 191.25 & 191 & 191 & 191 & 191 & 191 & 192 \\
\hline $\max$ & 255 & 255 & 255 & 255 & 255 & 255 & 255 \\
\hline Key Space & $2^{320}$ & $2^{405}$ & $2^{448}$ & $2^{2048}$ & $2^{256}$ & $2^{2183}$ & $2^{407}$ \\
\hline $\begin{array}{l}\text { Key Sensitivity } \\
\text { UACI } \\
\text { NPCR }\end{array}$ & $\begin{array}{l}0.336 \\
0.997\end{array}$ & $\begin{array}{l}0.3352 \\
0.9965\end{array}$ & $\begin{array}{l}0.3346 \\
0.99673\end{array}$ & $\begin{array}{l}0.3345 \\
0.9961\end{array}$ & $\begin{array}{l}0.3348 \\
0.9960\end{array}$ & $\begin{array}{l}0.3338 \\
0.9966\end{array}$ & $\begin{array}{l}0.3337 \\
0.9964\end{array}$ \\
\hline $\begin{array}{l}\text { Plain Pixels Sensitivity } \\
\text { UACI } \\
\text { NPCR }\end{array}$ & $\begin{array}{l}0.331 \\
0.9964\end{array}$ & $\begin{array}{l}0.3312 \\
0.9958\end{array}$ & $\begin{array}{l}0.3253 \\
0.9954\end{array}$ & $\begin{array}{l}4 \mathrm{E}-6 \\
0.0001\end{array}$ & $\begin{array}{l}0.3339 \\
0.9959\end{array}$ & $\begin{array}{l}0.3342 \\
0.9951\end{array}$ & $\begin{array}{l}0.3317 \\
0.9961\end{array}$ \\
\hline PSNR (dB) & 10.45 & 7.733 & 9.43 & 7.749 & 7.740 & 9.731 & 9.153 \\
\hline Entropy & 7.9984 & 7.999 & 7.998 & 7.9998 & 7.999 & 7.9992 & 7.999 \\
\hline Horizontal Correlation & 0.0045 & $3.7 \mathrm{E}-5$ & $6 \mathrm{E}-4$ & $8.1 \mathrm{E}-4$ & $1.4 \mathrm{E}-3$ & $4.5 \mathrm{E}-3$ & -0.0485 \\
\hline Vertical Correlation & 0.0072 & $3.3 \mathrm{E}-5$ & 9E-4 & $2 \mathrm{E}-5$ & $1.5 \mathrm{E}-3$ & 0.0039 & 0.0643 \\
\hline Diagonal Correlation & 0.0025 & $3.6 \mathrm{E}-3$ & $3 \mathrm{E}-4$ & $1.2 \mathrm{E}-3$ & $4.5 \mathrm{E}-4$ & 0.0054 & 0.0035 \\
\hline FPS & 10.274 & 10.05 & 5.605 & 3.74 & 1.024 & 1.215 & 0.346 \\
\hline
\end{tabular}

[31] S. Ribaric, A. Ariyaeeinia, and N. Pavesic, "De-identification for privacy protection in multimedia content: A survey," Signal Processing: Image Communication, vol. 47, pp. 131-151, 2016.

[32] M. Saini, P. K. Atrey, S. Mehrotra, and M. Kankanhalli, "W 3-privacy: understanding what, when, and where inference channels in multicamera surveillance video," Multimedia Tools and Applications, vol. 68, no. 1, pp. 135-158, 2014

[33] Z. Tang, Y. Yang, S. Xu, C. Yu, and X. Zhang, "Image encryption with double spiral scans and chaotic maps," Security and Communication Networks, vol. 2019, 2019.

[34] T. Winkler and B. Rinner, "Security and privacy protection in visual sensor networks: A survey," ACM Computing Surveys (CSUR), vol. 47, no. 1, pp. 1-42, 2014

[35] S. Xiao, Z. Yu, and Y. Deng, "Design and analysis of a novel chaosbased image encryption algorithm via switch control mechanism," Security and Communication Networks, vol. 2020, 2020.

[36] R. Xu, S. Y. Nikouei, Y. Chen, E. Blasch, and A. Aved, "Blendmas: A blockchain-enabled decentralized microservices architecture for smart public safety," in 2019 IEEE International Conference on Blockchain (Blockchain). IEEE, 2019, pp. 564-571.

[37] R. Xu, S. Y. Nikouei, D. Nagothu, A. Fitwi, and Y. Chen, "Blendsps: A blockchain-enabled decentralized smart public safety system," Smart Cities, vol. 3, no. 3, pp. 928-951, 2020.

[38] L. Yuan and T. Ebrahimi, "Image privacy protection with secure jpeg transmorphing," IET Signal Processing, vol. 11, no. 9, pp. 1031-1038, 2017.

[39] M. Yuan, S. Y. Nikouei, A. Fitwi, Y. Chen, and Y. Dong, "Minor privacy protection through real-time video processing at the edge," arXiv preprint arXiv:2005.01178, 2020.

[40] X. Zhang and X. Wang, "Chaos-based partial encryption of spiht coded color images," Signal Processing, vol. 93, no. 9, pp. 2422-2431, 2013.

[41] Y. Zhou, L. Bao, and C. P. Chen, "A new 1d chaotic system for image encryption,” Signal processing, vol. 97, pp. 172-182, 2014.

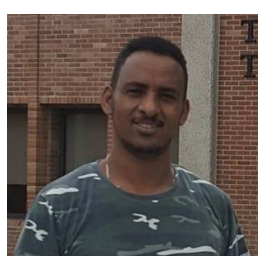

Alem Fitwi is a Ph.D. candidate of the Dept. of Electrical and Computer Engineering at Binghamton University - State University of New York (SUNY). His research area includes Privacy-and-Security, Edge Surveillance, Machine/Deep Learning, and Blockchain.

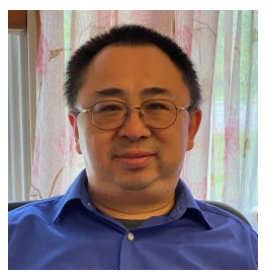

Yu Chen is an Associate Professor at the Dept. of Electrical and Computer Engineering, Binghamton University - SUNY. He received the Ph.D. in Electrical Engineering from the University of Southern California (USC) in 2006. His research interest lies in Trust, Security and Privacy in Computer Networks, including Edge-Fog-Cloud Computing, Internet of Things (IoT), and their applications in smart and connected environments. His publications include over 150 papers in scholarly journals, conference proceedings, and books.

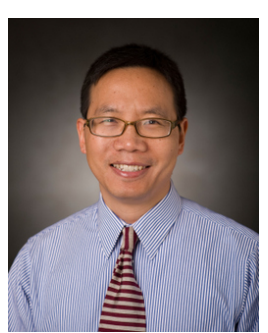

Sencun Zhu received the B.S. degree in precision instruments from Tsinghua University, China, in 1996, the M.S. degree in signal processing from the University of Science and Technology of China, Graduate School at Beijing, China, in 1999, and the Ph.D. degree in information technology from George Mason University, USA, in 2004. He is an Associate Professor with Penn State University. His research interests focus on mobile and wireless security and privacy. He is the co-editor-in-chief of EAI Transactions on Security and Safety, an associate editor of IEEE Transactions on Mobile Computing (TMC) and Wiley Journal on Security and Privacy. 